\title{
Generation of Second Harmonics of Relativistically Self-Focused q-Gaussian Laser Beams in Underdense Plasma with Axial Density Ramp
}

Naveen Gupta ( $\square$ naveen.21559@lpu.co.in )

Lovely Professional University

Sandeep Kumar

Lovely Professional University

\section{Research Article}

Keywords: q-Gaussian, Density Ramp, Self Focusing, Second Harmonic Generation.

Posted Date: February 22nd, 2021

DOI: https://doi.org/10.21203/rs.3.rs-163164/v1

License: (c) (1) This work is licensed under a Creative Commons Attribution 4.0 International License.

Read Full License

Version of Record: A version of this preprint was published at Optical and Quantum Electronics on April 3rd, 2021. See the published version at https://doi.org/10.1007/s11082-021-02827-w. 


\title{
Generation of Second Harmonics of Relativistically Self-Focused q-Gaussian Laser Beams in Underdense Plasma with Axial Density Ramp
}

\author{
Naveen Gupta and Sandeep Kumar \\ Lovely Professional University, Phagwara \\ India \\ Email :- naveens222@rediffmail.com
}

\begin{abstract}
An investigation on frequency doubling of intense laser beams through the phenomenon of second harmonic generation (SHG) in underdense plasmas has been presented. In order to increase the efficiency of S.H.G the density profile of plasma has been considered in the shape of upward ramp. When laser beam with frequency $\omega_{0}$ propagates through plasma, it makes the plasma electrons to oscillate at pump frequency. These oscillations of plasma electrons in the presence of thermal velocity generate a plasma wave at frequency $\omega_{0}$. The generated plasma wave beats with the pump beam to double its frequency. Variational theory has been adopted to find semi analytical solution of the wave equation for the slowly varying envelope of the laser beam. By using hydrodynamic fluid model of plasma, nonlinear current density for SHG has been obtained. Emphasis are put on investigation of the effect of various laser and plasma parameters on propagation dynamics of pump beam and the power of generated second harmonics.
\end{abstract}

\section{Key Words}

q-Gaussian, Density Ramp, Self Focusing, Second Harmonic Generation. 


\section{INTRODUCTION}

The quest for short wavelength coherent radiation sources has a long history. Coherent short wavelength radiations are the back bone for a broad variety of applications in various fields of basic and applied sciences[1]. Among them the most important are femto second time resolved coherent diffractive imaging[2] and photo induced processes at surfaces and nanoparticles[3], lithography[4], plasma diagnostics[5], and material processing and diagnostics[6] etc. For past few decades, only two main facilities, namely free electron lasers[7] and synchrotrons[8] are being considered for this purpose. However, due to their huge infrastructure the access to these facilities is limited. Therefore, research associated with these facilities is not growing at a faster rate. In contrast, coherent short wavelength sources based on laser harmonics[9,10] can be accommodated in a table top device and are therefore cost effective alternate to free electron lasers and synchrotrons. Thus the phenomenon of higher harmonic generation (HHG) bears the promise to bring coherent radiation sources within the reach of universities and hospitals. Along with steady improvements in size and cost, the unique characteristics of laser driven harmonic sources are expected to be useful for a wide range of contests, including radiation therapy for treatment of cancers, material characterization, detection of narcotics and other dangerous substances. Thus, laser driven radiation sources based on HHG can adieu to the era of gargantuan synchrotrons and thus will push physicists towards the era of bench top coherent radiation sources that could revolutionize cancer diagnostics and treatment.

Laser harmonics can be produced in a number of nonlinear media like gases[11], dielectrics[12], semiconductors[13] and plasmas[14-17]. In comparison to normal states of matter (i.e., solids, liquids and gases), plasmas are having several interesting advantages that make them ideal candidate for the generation of laser harmonics. Firstly, being already ionized, plasmas are 
immune to material damage. Hence, there is no upper limit on the intensity of pump beam that can be sent through the plasmas. Secondly, harmonic generation in plasmas is associated with free electrons and hence, laser harmonics of any order can be produced in plasmas.

There are a number of mechanisms by which laser harmonics can be produced in plasmas. This includes parametric instabilities[18], resonance absorption[19], ionization absorption[20], photon acceleration[21], density gradients associated with excitation of EPW[22]. In case of SHG, the main mechanism is excitation of an electron plasma wave (EPW) at pump frequency by the incident laser beam. The nonlinear coupling of electron density perturbation associated with EPW to the pump beam results in the generation of an electromagnetic beam at frequency double of pump beam.

The phenomenon of SHG was first reported by Franken[12] in 1961 immediately after the invention of laser. Since then, a number of investigations highlighting theoretical as well as experimental aspects of SHG have been reported by several researchers. However, from literature review it has been seen that in previous investigations on SHG of laser beams in plasmas with density ramped density profile, it has been assumed that the irradiance over the beam's cross section is having ideal Gaussian profile. However, experimental investigations[24] on the irradiance profile of ultra intense laser systems such as those are involved in inertial confinement fusion indicate that although the laser is operating in its fundamental mode i.e., TEM $_{00}$ mode, its irradiance profile is not exactly Gaussian. In comparison to ideal Gaussian profile the wings of the irradiance profile of the actual laser system are fond to be expanded radially i.e., a significant amount of laser energy lies in off axial region of the cross section. By fitting into experimental data[25] it has been suggested that the actual irradiance profile of the laser beam can be modeled by Tsalli's $q$-Gaussian distribution[26]. Since, no earlier theoretical investigation on SHG of $q$-Gaussian laser beams in plasmas with density ramp has been reported 
till date, this gave us strong motivation to investigate the same. Thus, this paper aims to present first theoretical investigation on SHG of $q$-Gaussian laser beam in underdense plasma with density ramp.

\section{Relativistic Nonlinearity of Plasma}

The dielectric function of a plasma whose electron density increases axially as $n(z)=n_{0}(1+$ $\tan (b z))$ can be written as $[27]$

$$
\epsilon=1-\frac{4 \pi e^{2} n_{0}}{m_{e} \omega_{0}^{2}}(1+\tan (a z))
$$

where, $e$ and $m_{e}$ are the electronic charge and mass respectively, $n_{0}$ is the equilibrium electron density at $z=0$ and the constant $b$ describes the rate of change of electron density with distance. When a laser beam with electric filed vector

$$
\mathbf{E}(r, z, t)=A_{0}(r, z) e^{\iota\left(k_{0} z-\omega_{0} t\right)} \mathbf{e}_{\mathbf{x}}
$$

propagates through such a plasma target, it makes the plasma electrons to oscillate at velocity

$$
\mathbf{v}=-\iota \frac{e}{\omega_{0} m_{e}} \mathbf{E}
$$

where, $\omega_{0}$ is the angular frequency of the laser beam. If the amplitude of the electric filed of the laser beam is sufficiently large, then the quiver velocity of plasma electrons becomes comparable to that of light in vacuum. Hence, during the interaction of ultra intense laser beam with plasma, the plasma electrons experience a relativistic increase in their mass. Hence, the effective mass $m_{e}$ of plasma electrons in eq.(1) need to be replaced by $m_{e}=m_{0} \gamma$, where $m_{0}$ is the rest mass of electron and $\gamma$ is the relativistic Lorentz factor. Following Akhiezer and Polovin $\gamma$ is related to laser amplitude as[28]

$$
\gamma=\left(1+\beta A_{0} A_{0}^{\star}\right)^{\frac{1}{2}}
$$


where, $\beta=\frac{e^{2}}{m_{0}^{2} c^{2} \omega_{0}^{2}}$ is the constant associated with the strength of relativistic nonlinearity and is termed as coefficient of relativistic nonlinearity. Hence, in the presence of laser beam eq.(1) gets modified as

$$
\epsilon=1-\frac{\omega_{p 0}^{2}}{\omega_{0}^{2}}\left(1+\beta A_{0} A_{0}^{\star}\right)^{-\frac{1}{2}}(1+\tan (b z))
$$

where, $\omega_{p 0}=\sqrt{\frac{4 \pi e^{2}}{m_{0}} n_{0}}$ is the plasma frequency in the absence of laser beam. Writing the effective dielectric function of plasma as sum of linear $\left(\epsilon_{0}\right)$ and nonlinear $\phi$ parts as

$$
\epsilon=\epsilon_{0}+\phi\left(A_{0} A_{0}^{\star}\right)
$$

we get

$$
\epsilon_{0}=1-\frac{\omega_{p 0}^{2}}{\omega_{0}^{2}}
$$

and

$$
\phi\left(A_{0} A_{0}^{\star}\right)=\frac{\omega_{p 0}^{2}}{\omega_{0}^{2}}\left(1-\frac{1}{\left(1+\beta A_{0} A_{0}^{\star}\right)^{\frac{1}{2}}}\right)(1+\tan (a z))
$$

Eq.(6) gives the relativistic nonlinear dielectric function of plasma.

\section{Dynamics of Pump Beam}

Laser beam propagation through a nonlinear medium is modeled by wave equation (that can be obtained from Maxwell's equations)[29]

$$
2 \iota k_{0} \frac{\partial A_{0}}{\partial z}=\nabla_{\perp}^{2} A_{0}+\frac{\omega_{0}^{2}}{c^{2}} \phi\left(A_{0} A_{0}^{\star}\right) A_{0}
$$

Eq.(7) is very much identical Schrodinger equation encountered in quantum mechanics. The only difference being that here time coordinate 't' has been replaced with space coordinate 'z' 
and here the potential function $\phi$ itself is dependent on the field amplitude $A_{0}$. Hence eq.(7) is also known as nonlinear Schrodinger wave equation (NLSE).

Being nonlinear in nature, superposition principle does not apply to eq.(7) i.e., linear combination of two solutions is not a solution. Mathematically this means that conventional method of solving partial differential equations i.e., expansion in power series are not applicable to NSWE. In fact no exact analytical solution exists for this equation. In order to obtain physical insight into the propagation dynamics of the laser beam we use a semi analytical technique known as variational method[30]. This method converts the problem of solving a partial differential equation to that of solving a set of coupled ordinary differential equations. These ordinary differential equations govern the evolution of the various parameters of interest. In case of self focusing of laser beams the parameter of interest is the beam width of the laser beam. According to this method eq.(7) is a variational problem for action principle based on Lagrangian density

$$
\mathcal{L}=\iota\left(A_{0} \frac{\partial A_{0}^{\star}}{\partial z}-A_{0}^{\star} \frac{\partial A_{0}}{\partial z}\right)+\left|\nabla_{\perp} A_{0}\right|^{2}-\frac{\omega_{0}^{2}}{c^{2}} \int^{A_{0} A_{0}^{\star}} \phi\left(A_{0} A_{0}^{\star}\right) d\left(A_{0} A_{0}^{\star}\right)
$$

In the present investigation the functional form of $A_{0}(r, z)$ has been taken as[15]

$$
A_{0}(r, z)=\frac{E_{00}}{f}\left(1+\frac{r^{2}}{q r_{0}^{2} f^{2}}\right)^{-\frac{q}{2}}
$$

where, phenomenological parameter $q$ is related to the deviation of irradiance, over the cross section of the beam, from ideal Gaussian profile and is therefore termed as deviation parameter. The function $f(z)$ upon multiplication with initial beam width $r_{0}$ it gives the instantaneous spot size (i.e., spot size at particular location inside the medium) of the laser beam. Hence, the function $f(z)$ is referred to as dimensionless beam width parameter.

In order to see the effect of deviation parameter ' $q^{\prime}$ on the distribution of intensity over the cross section of the laser beam, we have portrait the normalized intensity vs radial coordinate 
curves for different value of $q$ in fig.(1). It can be seen that $q$ value of the laser beam does not affect the irradiance in the paraxial region of the beam's cross section. However, change in the $q$ value significantly affects the irradiance in the off axial region. With increase in the $q$ value, the irradiance in the off axial part of the cross section falls more rapidly, in the off axial part in comparison to that of the beams with lower $q$ values. In other words, increase the $q$ value increases the intensity gradient in the off axial part and hence makes the off axial part of the beam more weaker.

For $q \rightarrow \infty$, the amplitude structure of the laser beam gets reduced to ideal Gaussian i.e.,

$$
\lim _{q \rightarrow \infty} A_{0}(r, z)=\frac{E_{00}}{f} e^{-\frac{r^{2}}{2 r_{0}^{2} f^{2}}}
$$

Further in order to see the effect of parameter $q$ on the initial beam width of the laser beam, we make use of spatial intensity moments of the laser beam. In this regard the effective beam width of the laser beam is defined in r.m.s sense as

$$
<a^{2}>=\frac{1}{I_{0}} \iint A_{0} r^{2} A_{0}^{\star} d^{2} r
$$

with

$$
\begin{gathered}
I_{0}=\iint A_{0} A_{0}^{\star} d^{2} r \\
d^{2} r=r d r d \theta
\end{gathered}
$$

Hence, for the intensity profile given by eq(9), the value of effective laser beam width has been obtained as

$$
<a^{2}>=r_{0}^{2} f^{2}\left(1-\frac{2}{q}\right)^{-1}
$$

Variation of normalized beam width of $q$-Gaussian laser beam with $q$ (Fig.2) at the plane of incidence i.e., $z=0$, indicates that the effect of increase in the value of $q$ is to reduce the 
effective beam width of the beam. As the divergence of an optical beam varies inversely with its effective beam width, it can be predicted from eq.(10) (fig.2) that ideal Gaussian beams $(q \rightarrow \infty)$ possess maximum diffraction. This fact will be further validated by solving the wave equation for the beam envelope in vacuum (fig.3).

Substituting the trial function (eq.9) in lagrangian density and integrating over $r$ we get reduced Lagrangian as $L=\int_{0}^{\infty} \mathcal{L} r d r$. The corresponding Euler-Lagrange equation

$$
\frac{d}{d z}\left(\frac{\partial L}{\partial\left(\frac{\partial f}{\partial z}\right)}\right)-\frac{\partial L}{\partial f}=0
$$

gives following ordinary differential equation for the beam width of the laser beam:

$$
\frac{d^{2} f}{d \xi^{2}}=\frac{\left(1-\frac{1}{q}\right)\left(1-\frac{2}{q}\right)}{\left(1+\frac{1}{q}\right)} \frac{1}{f^{3}}-\left(\frac{\omega_{p 0} r_{0}}{c}\right)^{2}(1+\tan (d \xi))\left(1-\frac{1}{q}\right)\left(1-\frac{2}{q}\right) \frac{\beta E_{00}^{2}}{f^{3}} T
$$

where,

$$
\begin{gathered}
T=\int_{0}^{\infty} x\left(1+\frac{x}{q}\right)^{-2 q-1}\left(1+\frac{\beta E_{00}^{2}}{f^{2}}\left(1+\frac{x}{q}\right)^{-q}\right)^{-\frac{3}{2}} d x \\
x=\frac{r^{2}}{r_{0}^{2} f^{2}} \\
d=b k_{0} r_{0}^{2} \\
\xi=\frac{z}{k_{0} r_{0}^{2}}
\end{gathered}
$$

In the present investigation eq.(12) has been solved numerically with the help of Runge Kutta fourth order technique under the assumption that while entering into the plasma medium the beam is collimated and is having plane wavefront. This imposes the boundary equations $f=1$ and $\frac{d f}{d \xi}=0$ at $\xi=0$ on eq.(12).

The variation of normalized density $\frac{n(\xi)}{n_{0}}$ with longitudinal distance $\xi$ for different values of $d$ has been shown in fig.3. It can be seen that the effect of parameter $d$ is to increase the slope of the ramp i.e., to increase the rate of increase of plasma density with distance. 
Firstly, in order see the linear propagation of $q$-Gaussian laser beam, eq.(12) has been solved in the absence of plasma medium and the corresponding variations of the beam width have been shown in fig.4. It can be seen that whatever is the value of $q$ the laser beam diffracts in vacuum. This is a consequence of the light's inherent property to diffract. An interesting result is that as the beam profile converges to Gaussian, the diffraction of the laser beam becomes stronger. This fact can be explained by examining the initial beam profile (fig.1) or the effect of $q$ on initial beam width (fig.2). It can been seen from fig.2 that laser beams whose intensity distribution is much closer to Gaussian one possess smaller effect beam with. As the diffraction broadening of the optical beam varies inversely as its beam width, increase in $q$ value makes the diffraction of the laser beam stronger.

Further, in order to see the evolution of beam envelope through the plasma, eq. 8 has been solved for:

$$
\omega_{0}=1.78 \times 10^{14} \mathrm{rad} / \mathrm{sec}, r_{0}=10 \mu \mathrm{m}, \frac{\omega_{p 0}^{2} r_{0}^{2}}{c^{2}}=12
$$

The corresponding evolution of the beam width with distance through the plasma is shown in fig.(5). It can be seen that during the propagation of laser beam through plasma, its beam width varies harmonically with distance. This behaviour of the beam width can be explained by examining the role and origin of various terms contained in eq.(12). The first term on right hand side (R.H.S), which is proportional to $f^{-3}$, is the spatial dispersive term, that models the spreading of the laser beam in transverse directions occurring as a consequence of diffraction divergence. The second term which is having complex dependence on $f$, arises due relativistic mass nonlinearity of the carriers and is responsible for nonlinear optical response of the plasma to pump beam. As a consequence of this optical nonlinearity of the plasma the resulting nonlinear refraction of the laser beam tends to counter balance the effect of 
diffraction. Thus, during the propagation of the laser beam through plasma, there starts a competition between the two phenomena of diffraction and nonlinear refraction. The winning phenomenon ultimately decides the behaviour of the laser beam i.e., whether the beam will converge or diverge. Thus, there exists a critical value of beam intensity (that can be obtained by balancing the two terms on the right hand side of eq.(12) above which the beam will converge. In the present investigation the initial beam intensity has been taken greater than the critical intensity i.e., why the laser spot size of the laser beam is converging initially. As the beam width decreases, its intensity increases. When the laser intensity becomes too high the mass of plasma electrons in the illuminated portion of plasma gets saturated and thus the nonlinear refraction vanishes, leaving only the diffraction effects to dominate. Hence, after focusing to minimum, the beam width bounces back to its original value. As the beam width of the laser beam starts increasing, the competition between diffraction and nonlinear refraction starts again. Now, this competition lasts till maximum value of $f$ is obtained. These processes go on repeating themselves and thus give breather like behaviour to the spot size of the laser beam.

Further it has been observed that after every focal spot the maximum as well as the minimum of the beam width shift downwards. This is owing to the fact that the equilibrium electron density is an increasing function of longitudinal distance. Hence, the plasma index of refraction keep on decreasing with the penetration of laser beam into the plasma. Consequently, the self focusing effect gets enhanced and the maximum as well as minimum of the beam width go on shifting downwards after every focal spot. It is also seen that the frequency of oscillations of beam with increases with distance. The physics behind this fact is that denser is the plasma, higher will be the phase velocity of laser beam through it. Hence, in denser plasma laser beam takes less duration to get self focused.

Reduced focusing of the laser beams with higher value of $q$ can also be seen from fig.5. The 
reason behind this effect is that for laser beams with higher value of $q$ most of the intensity is concentrated in a narrow region around the axis of the beam. Hence, off axial part of these beams do not give significant contribution in order to produce nonlinearity in the medium. As the phenomenon of self focusing is a homeostasis of nonlinear refraction of the laser beam due to optical nonlinearity of the medium, increase in the value of $q$ reduces the extent of self focusing.

The plots in fig.5 also indicate that instead of their reduced focusing, the laser beams with higher value of $q$ possess faster focusing character. This is due to the faster focusing character of axial rays. Being away from the axis, off axial rays take more duration to get self focused. As there are more number of off axial rays in laser beams with lower $q$ values, hence with increase in the value of $q$, the focusing of the laser beam becomes faster. Hence, by optimizing the value of $q$, one can control the extent of self focusing as well as the location of the focal spot of a laser beam.

Fig.6 illustrates the effect of initial beam intensity on self focusing of the laser beam. It can be seen that with increase in the initial intensity of the laser beam, there is enhancement in the extent of self focusing of the laser beam and also it becomes faster. This occurs because with increase in the intensity of pump beam, the relativistic effect becomes stronger that in turn increases the transverse gradient of the index of refraction of the illuminated portion of plasma. Thus increase in the laser intensity enhances the amount of focusing of the laser beam.

Fig.7 illustrates the effect of slope of the density ramp on self focusing of the laser beam. It can be seen that with increase in the slope of density ramp, there is enhancement in the extent of self focusing of the laser beam. This occurs because with increase in the slope of the density ramp, in the deeper regions of the plasma the laser beam sees lesser index of refraction. Thus, increase in the slope of density ramp enhances the extent of focusing of the laser beam. 


\section{Generation of Second Harmonics}

The nonuniform irradiance over the cross section of the laser beam produces a transverse density gradient in the plasma by evacuating electrons from high intensity regions. These density gradients result in an EPW at pump frequency $\omega_{0}$, whose amplitude is governed by wave equation

$$
-\omega_{0}^{2} n^{\prime}+v_{t h}^{2} \nabla^{2} n^{\prime}+\omega_{p}^{2}(\xi) n^{\prime}=\frac{e}{m} n_{0}(1+\tan (d \xi)) \nabla A_{0}
$$

By taking $n^{\prime}=n^{\prime \prime} e^{\iota\left(\omega_{0} t-k_{0} z\right)}$ we get the amplitude of plasma wave as

$n^{\prime \prime}=\frac{e n_{0}}{m}(1+\tan (d \xi)) \frac{E_{00}}{r_{0}^{2} f^{3}} \frac{r}{\left(\omega_{0}^{2}-k_{0}^{2} v_{t h}^{2}-\omega_{p 0}^{2}(1+\tan d \xi)\left\{1+\frac{E_{00}^{2}}{f^{2}}\left(1+\frac{r^{2}}{q r_{0}^{2} f^{2}}\right)^{-q}\right)\right\}^{-\frac{1}{2}}}\left(1+\frac{r^{2}}{q r_{0}^{2} f^{2}}\right)^{-\frac{q}{2}-1}(14)$

The generated EPW interacts nonlinearly with the incident beam to produce its second harmonics. The electric field vector $\mathbf{E}_{\mathbf{2}}$ of the second harmonics satisfies the wave equation[31-33]

$$
\nabla^{2} \mathbf{E}_{\mathbf{2}}+\frac{\omega_{2}^{2}}{c^{2}} \epsilon_{2}\left(\omega_{2}\right) \mathbf{E}_{\mathbf{2}}=\frac{4 \pi e^{2}}{c^{2}} n^{\prime \prime} A_{0}
$$

that gives

$$
E_{2}=\frac{4 \pi e^{2}}{m} n^{\prime} \frac{A_{0}}{\left(k_{2}^{2}-4 k_{0}^{2}\right)}
$$

Defining the normalized power $P$ of second harmonics as

$$
P=\frac{P_{2}}{P_{0}}
$$

where,

$$
\begin{aligned}
& P_{2}=\int_{0}^{\infty} E_{2} E_{2}^{\star} r d r \\
& P_{0}=\int_{0}^{\infty} A_{0} A_{0}^{\star} r d r
\end{aligned}
$$


we get

$$
\begin{gathered}
P=\frac{8}{9} \frac{K_{0} T_{0}}{m c^{2}}\left(\frac{\omega_{0}^{2} r_{0}^{2}}{c^{2}}\right) \frac{\beta E_{00}^{2}}{f^{4}} H \\
H=\int_{0}^{\infty} \frac{\left(1+\frac{x}{q}\right)^{-2 q-2}}{\left(\left(\frac{\omega_{0}^{2} r_{0}^{2}}{c^{2}}\right)-\frac{\omega_{0}^{2} r_{0}^{2}}{c^{2}} \frac{\epsilon_{0} v_{t h}^{2}}{c^{2}}-\frac{\omega_{p 0}^{2} r_{0}^{2}}{c^{2}}\left(1+\tan \left(\frac{\xi}{d}\right)\right)\left\{1+\frac{\beta E_{00}^{2}}{f^{2}}\left(1+\frac{x}{q}\right)^{-q}\right\}^{-\frac{1}{2}}\right)^{2}} x d x
\end{gathered}
$$

Eq.(18) governs the evolution of second harmonic power $P$ with distance of propagation. This equation has been solved numerically in association with eq.(12) for plasma temperature $T_{0}=$ $10^{6} \mathrm{~K}$ in order to envision how the power of generated second harmonics evolve with longitudinal distance through the plasma. The corresponding variation of $P$ with distance $\xi$ has been depicted in fig.8. It has been observed that the power of second harmonic radiation is a monotonically increasing function of propagation, showing step like behaviour. Each step occurs at the position of the minimum beam width. This is because as the pump beam gets self focused its intensity increases and consequently the oscillation amplitude of the plasma electrons also increases which in turn increases the amplitude of the generated EPW. Since, the density perturbation associated with EPW acts as source for nonlinear current density for SHG, there is monotonic increase in the second harmonic power with distance.

The step like behaviour of the power of harmonic radiation at the positions of the minimum beam width of the pump beam is owing to the fact that these are the regions of highest intensity and hence the current density for SHG is maximum there. Hence, after attaining its maximum value the harmonic power moves towards its next possible higher value at next focal spot. These transitions of harmonic power form one maximum value to next maximum value give it a step like behaviour.

Further, it is observed that with longitudinal distance the steepness of the steps goes on increasing whereas the step size decreases. This is due the fact that steepness of steps is proportional to extent of self focusing and step size is inversely proportional to the frequency of 
oscillations of beam width. Since, with distance, the extent as ell as frequency of self focusing increase, these is increase in the steepness of the steps of $P$ vs. $\xi$ curve where as the size of steps goes on decreasing.

Reduction in the second harmonic power $P$ with increase in the value of deviation parameter $q$ has also been observed from the plots in fig.8. The underlying physics behind this is the one to one correspondence between the conversion to higher harmonics and the degree of self focusing of the pump beam. As increase in the value of $q$ reduces the extent of focusing of the pump beam, hence, there is corresponding decrease in the conversion efficiency of second harmonics.

The plots in fig.(9) and (10) indicate that the generated second harmonic power $P$ can be increased by increasing the initial intensity of the pump beam or the slope of density ramp. This is due to increase in the extent of self focusing with increase in intensity of the pump or slope of the density ramp.

\section{REFERENCES}

[1] Albert, F., Thomas, A. G. R., Mangles, S. P. D., Banerjee, S., et al. "Laser wakefield accelerator based light sources: potential applications and requirements." Plasma Physics and Controlled Fusion 56 084015(2014).

[2] Quiney, H.M., "Coherent diffractive imaging using short wavelength light sources." Journal of Modern Optics 571109 (2010).

[3] Li, D., Feng, J. and Pacifici, D., "Nanoscale optical interferometry with incoherent light." Scientific Rep. 61 (2016).

[4] Solak, H.H., "Nanolithography with coherent extreme ultraviolet light." Journal of physics D: Applied Phys. 39 R171 (2006). 
[5] Dobele, H.F., "Generation of coherent XUV radiation and its application to plasma diagnostics." Plasma Sources Science and Tech. 4224 (1995).

[6] Chen, Y., Fu, E., Yu, K., Song, M., Liu, Y., Wang, Y., Wang, H. and Zhang, X., "Enhanced radiation tolerance in immiscible $\mathrm{Cu} / \mathrm{Fe}$ multilayers with coherent and incoherent layer interfaces." J. Mat. Research 301300 (2015).

[7] McNeil, B.W. and Thompson, N.R., "X-ray free-electron lasers." Nature Photonics 4814 (2010).

[8] Bohon, J., D’Mello, R., Ralston, C., Gupta, S. and Chance, M.R., "Synchrotron X-ray footprinting on tour." J. Synchrotron Rad 21 24(2014).

[9] Teubner, U. and Gibbon, P., "High-order harmonics from laser-irradiated plasma surfaces," Rev. Mod. Phys. 81445 (2009).

[10] Winterfeldt, C., Spielmann, C. and Gerber, G., "Colloquium: Optimal control of highharmonic generation." Rev. Mod. Phys. 80117 (2008).

[11] Vampa, G. and Brabec, T., "Merge of high harmonic generation from gases and solids and its implications for attosecond science." J. Phys. B 50083001 (2017).

[12] Franken, E.P., Hill, A.E., Peters, C.W. and Weinreich, G., "Generation of optical harmonics." Phys. Rev. Lett. 7118 (1961).

[13] Orlando, G., Wang, C.M., Ho, T.S. and Chu, S.I., "High-order harmonic generation in disordered semiconductors." J. Opt. Soc. Am. B 35680 (2018). [

[14] Liu, C.S. and Tripathi, V.K., "Third harmonic generation of a short pulse laser in a plasma density ripple created by a machining beam.” Phys. Plasmas 15023106 (2008).

[15] Singh, A. and Gupta, N., "Higher harmonic generation by self-focused q-Gaussian laser beam in preformed collisionless plasma channel." Laser and Part. Beams 32621 (2014).

[16] Singh, R.P., Gupta, S.L. and Thareja, R.K., "Third harmonic generation in air ambient and 
laser ablated carbon plasma." Phys. Plasmas 22123302 (2015).

[17] Tyagi, Y., Tripathi, D. and Kumar, A., "Bernstein wave aided laser third harmonic generation in a plasma." Phys. Plasmas 23093115 (2016).

[18] Bobin, J.L., "High intensity laser plasma interaction." Phys. Rep. 122173 (1985).

[19] Erokhin, N.S., Zakharov, V.E. and Moiseev, S.S., "Second harmonic generation by an electromagnetic wave incident on inhomogeneous plasma." Sov. Phys. JETP 29101 (1969).

[20] Brunel, F., "Harmonic generation due to plasma effects in a gas undergoing multiphoton ionization in the high-intensity limit." JOSA B 7521 (1990).

[21] Wilks, S. C., Dawson, J. M., Mori, W. B., Katsouleas, T. and Jones, M. E., "Photon accelerator." Phys. Rev. Lett. 62, 2600 (1989).

[22] Parashar, J. and Pandey, H.D., "Second-harmonic generation of laser radiation in a plasma with a density ripple." IEEE Trans. Plasma Sci. 20996 (1992).

[23] Malka, V., Modena, A., Najmudin, Z., Dangor, A.E., Clayton, C.E., Marsh, K.A., Joshi, C., Danson, C., Neely, D. and Walsh, F.N., "Second harmonic generation and its interaction with relativistic plasma waves driven by forward Raman instability in underdense plasmas." Phys. Plasmas 41127 (1997).

[24] Nakatsutsumi, M., Davies, J.R., Kodama, R., Green, J.S., Lancaster, K.L., Akli, K.U., Beg, F.N., Chen, S.N., Clark, D., Freeman, R.R., Gregory, C.D., Habara, H., Heathcote, R., Hey, D.S., Highbarger, K., Jaanimagi, P., Key, M.H., Krushelnick, K., Ma, T., MacPhee, A., MacKinnon, A.J., Nakamura, H., Stephens, R.B., Storm, M.M., Tampo, Theobald, W., Woerkom, L.V., Weber, R.L., Wei, M.S., Woolsey, N.C. and Norreys, P.A., "Space and time resolved measurements of the heating of solids to ten million Kelvin by a petawatt laser." New J. Phys. 10, 043046 (2008).

[25] Patel, P.K., Key, M.H., Mackinnon, A.J., Berry, R., Borghesi, M., Chambers, D.M., Chen, 
H., Clarke, Damian, C., Eagleton, R., Freeman, R., Glenzer, S., Gregori, G., Heathcote, R., Hey, D., Izumi, N., Kar, S., King, J., Nikroo, A., Niles, A., Park, H.S., Pasley, J., Patel, N., Shepherd, R., Snavely, R.A., Steinman, D., Stoeckl, C., Storm, M., Theobald, W., Town, R., Maren, R.V., Wilks, S.C. and Zhang, B., "Integrated laser target interaction experiments on the RAL petawatt laser." Plasma Phys. Cont. Fusion 47, B833 (2005).

[26] Tsallis, C., "Possible generalization of Boltzmann-Gibbs statistics." J. Stat. Phys. 52, 479 (1988).

[27] Bokaei, B. and Niknam, A.R., "Increasing the upper-limit intensity and temperature range for thermal self-focusing of a laser beam by using plasma density ramp-up." Phys. Plasmas $\mathbf{2 1}$ 032309 (2014).

[28] Akhiezer, A.I. and Polovin, R.V., "Theory of wave motion of an electron plasma." Sov. Phys. JETP 3696 (1956).

[29] Gupta, N., "Self-action effects of quadruple-Gaussian laser beam in media possessing cubicquintic nonlinearity.” J. Electromagnetic Waves and Appl. 322350 (2018).

[30] Anderson, D., "Stationary Self-trapped Laser Beams in Plasma." Phys. Scripta 1835 (1978).

[31] Singh, K. P., Gupta, D. N., Yadav, S. and Tripathi, V. K., "Relativistic second-harmonic generation of a laser from underdense plasmas". Phys. Plasmas 12, 013101 (2005).

[32] Slaih, H. A. and Sharma, R. P., "Plasma wave and second-harmonic generation of intense laser beams due to relativistic effects". Phys. Plasmas 11, 3186 (2004).

[33] Purohit, G., "Second harmonic generation by self-focusing of intense hollow Gaussian laser beam in collisionless plasma". Phys. Plasmas 23, 013103 (2016). 


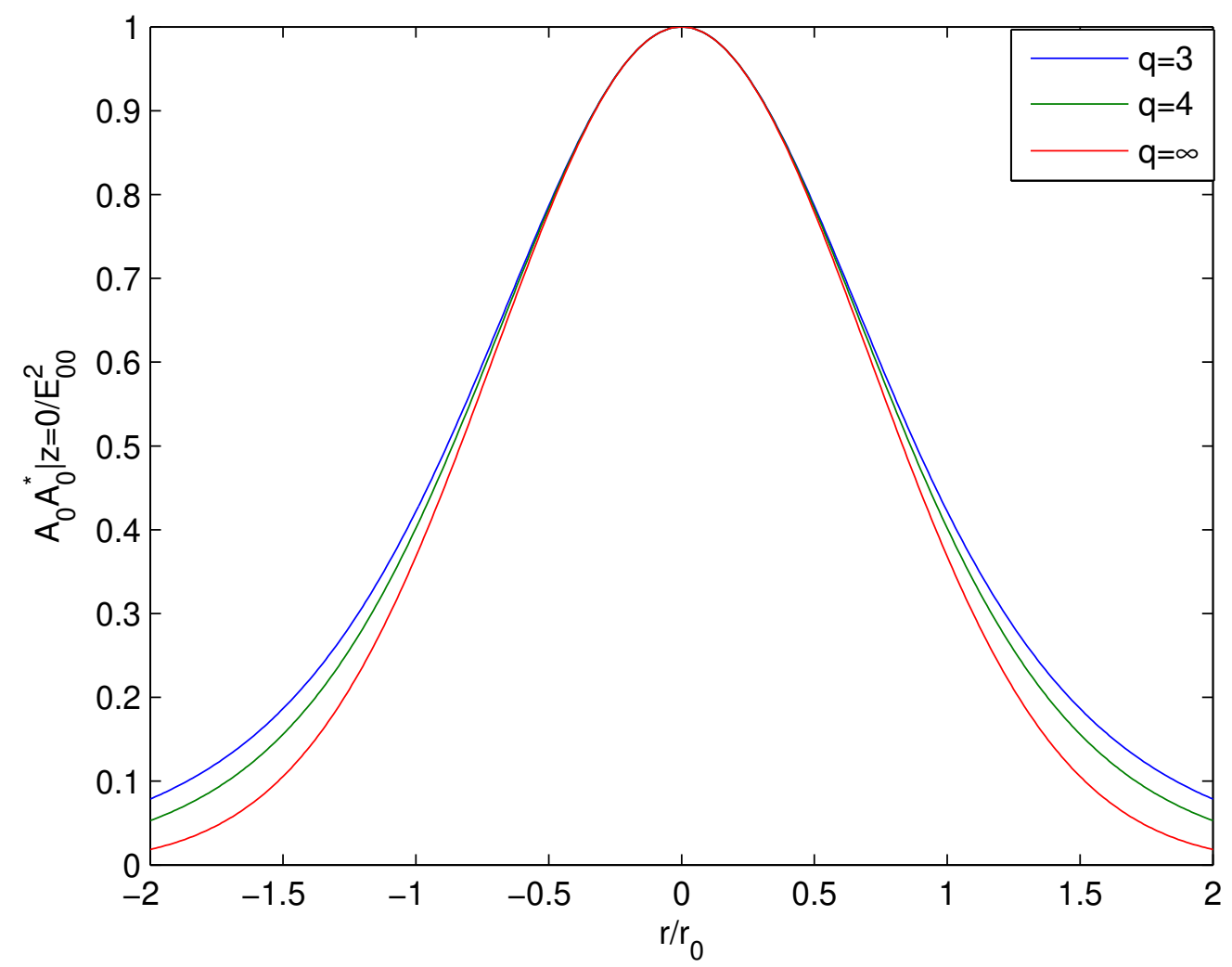

Figure 1: Effect of $q$ on irradiance over the cross section of the laser beam. 


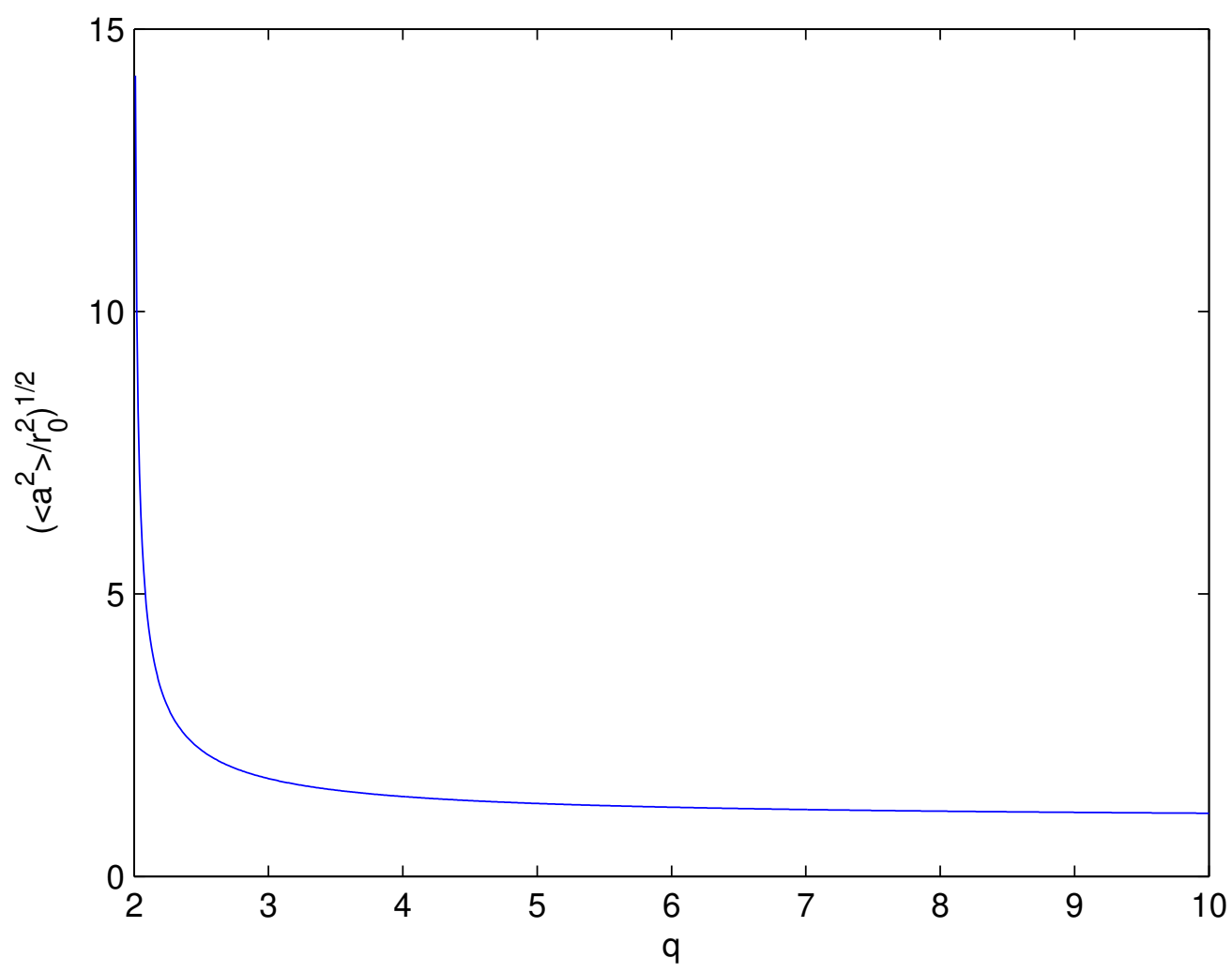

Figure 2: Variation of effective beam width of the laser beam with $q$.

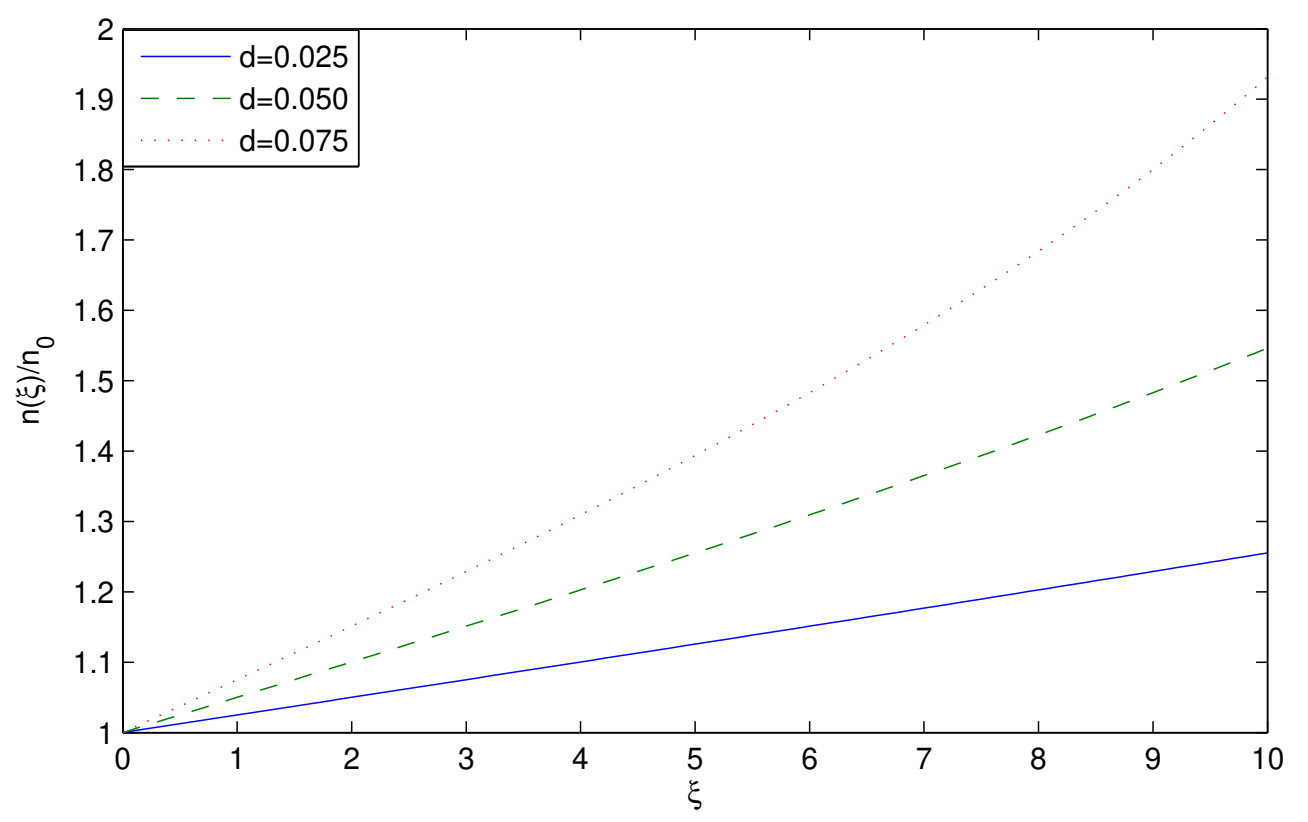

Figure 3: Effect of parameter d on equilibrium electro density with distance. 


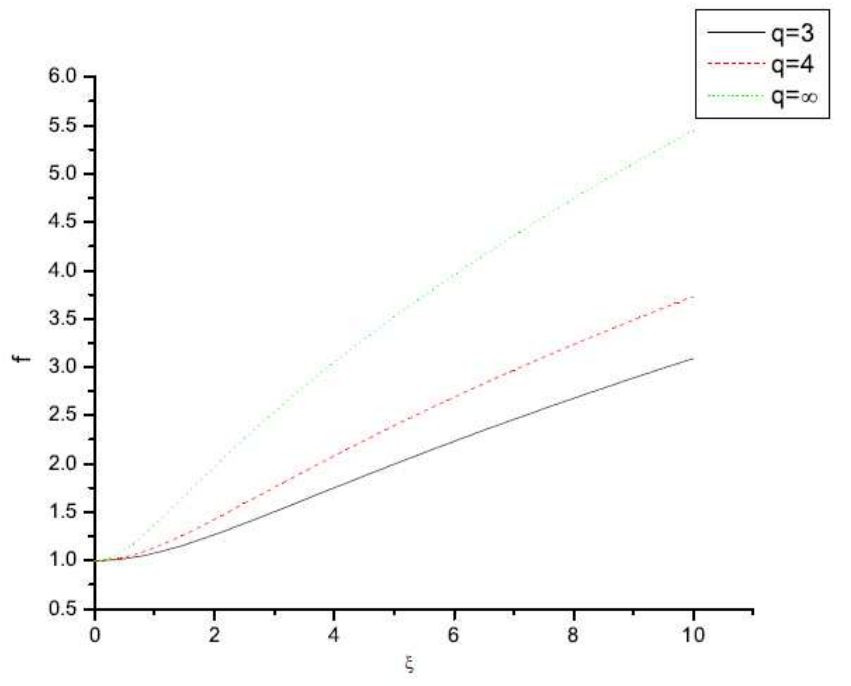

Figure 4: Effect of $q$ on vacuum diffraction of the laser beam.

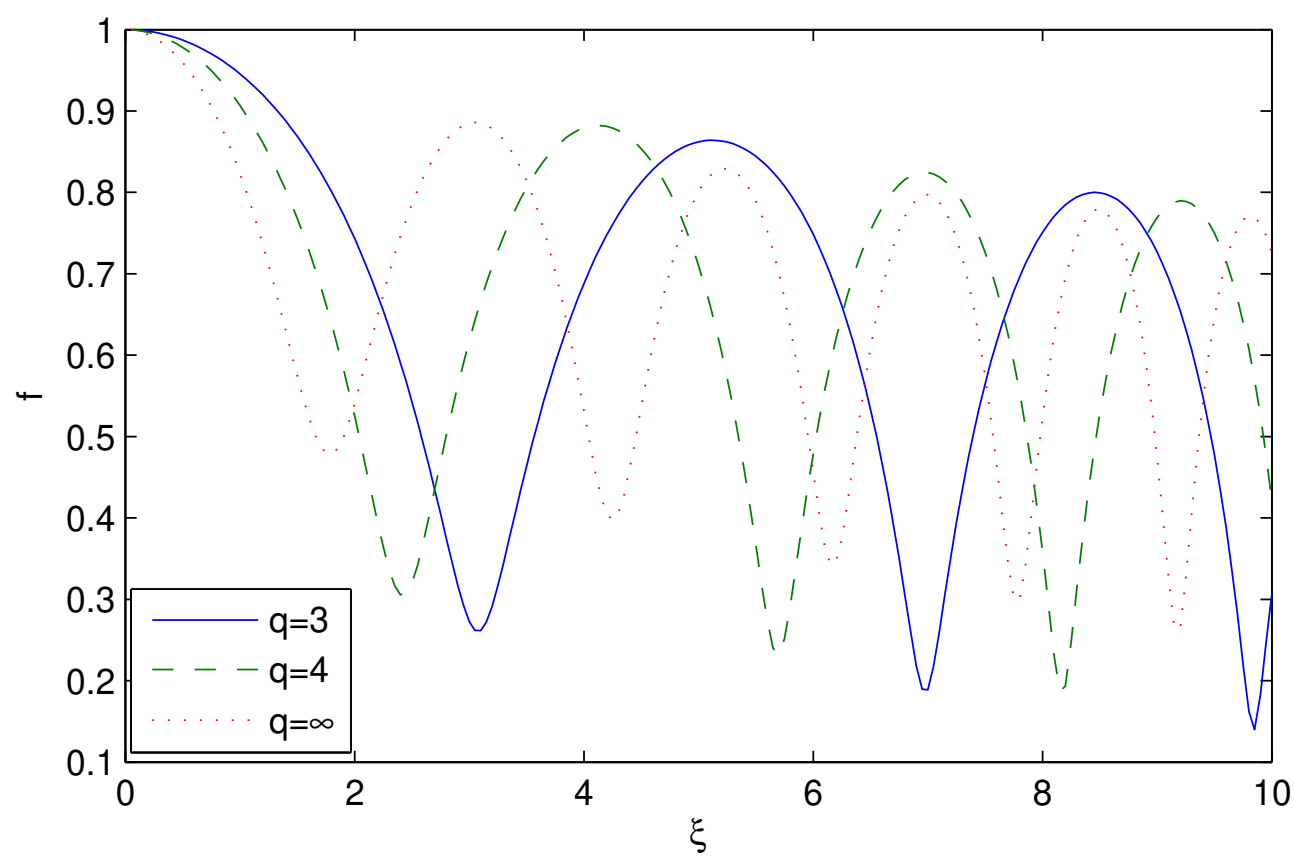

Figure 5: Effect of q on evolution of beam width in plasma density ramp. 


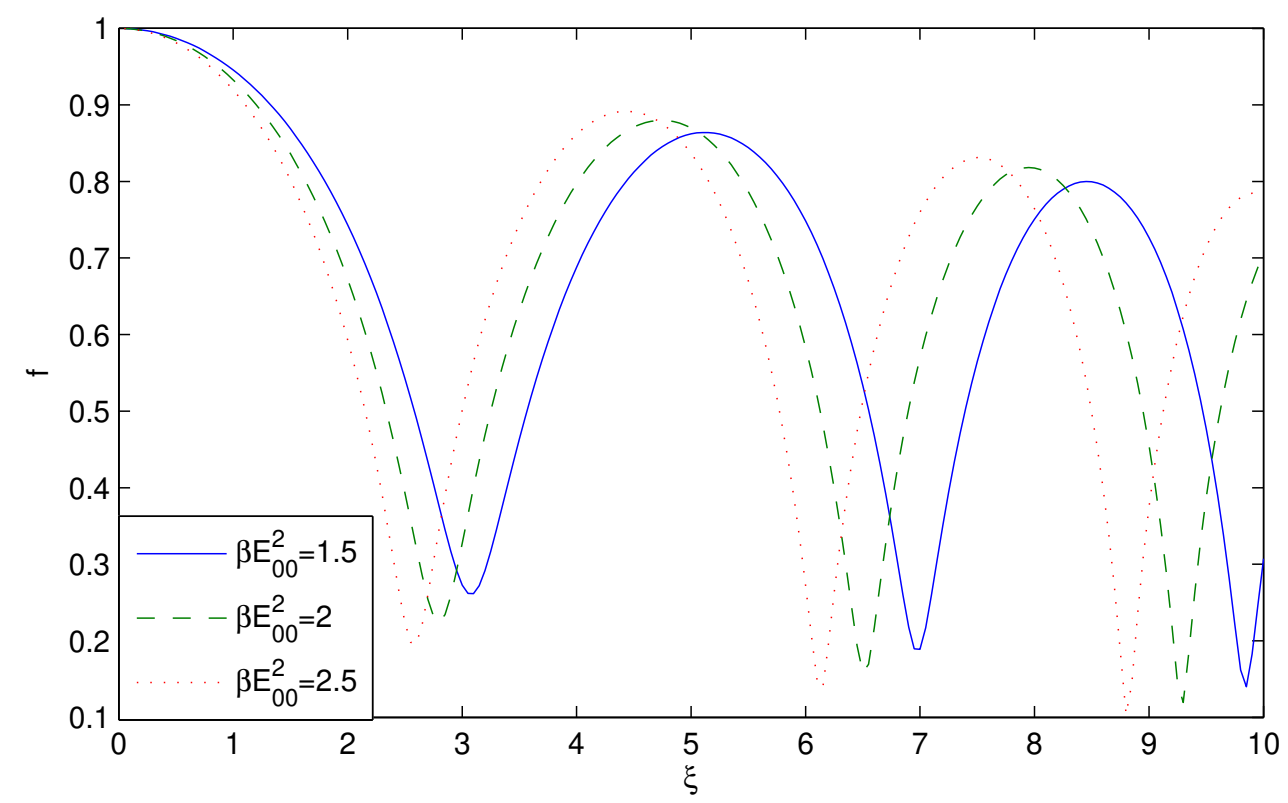

Figure 6: Effect of initial intensity of the laser beam on evolution of its beam width in plasma density ramp.

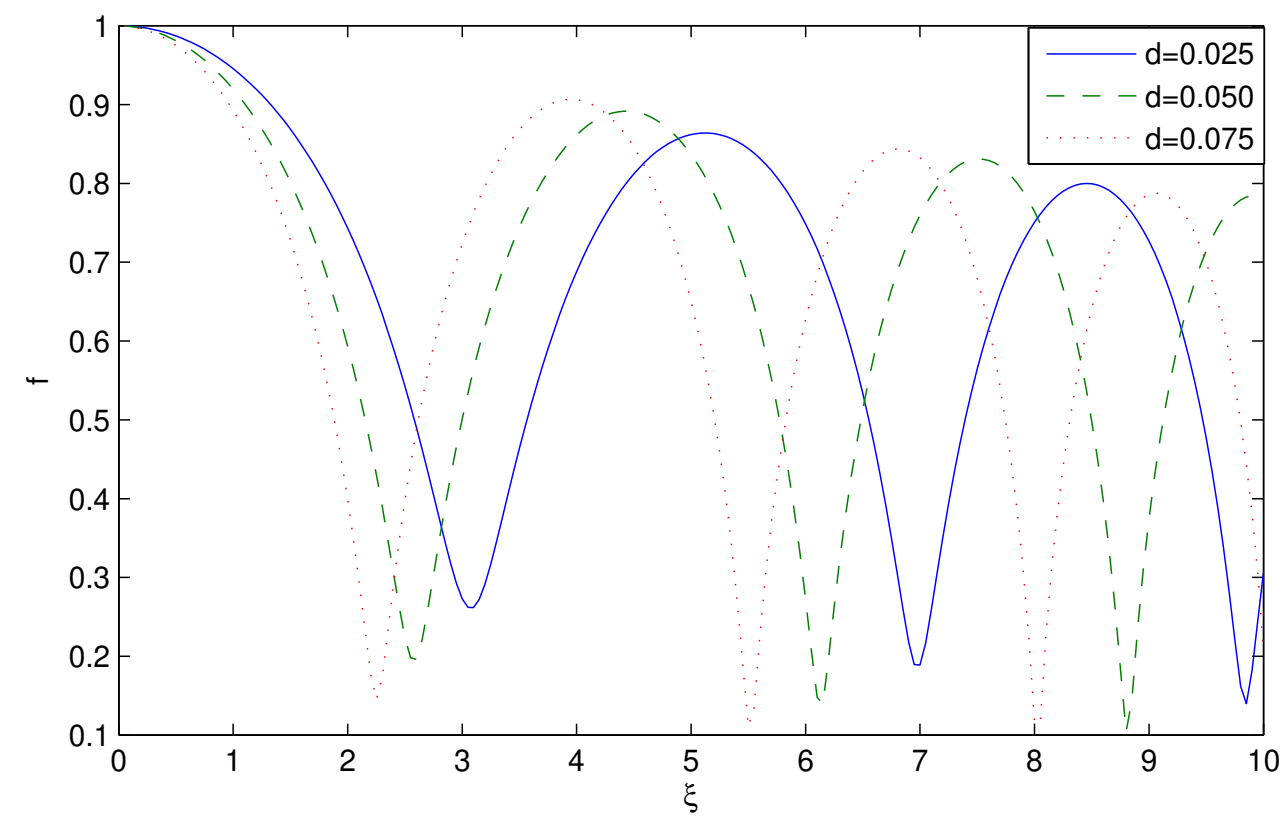

Figure 7: Effect of slope of the density ramp on evolution of beam width of the laser beam in plasma density ramp. 


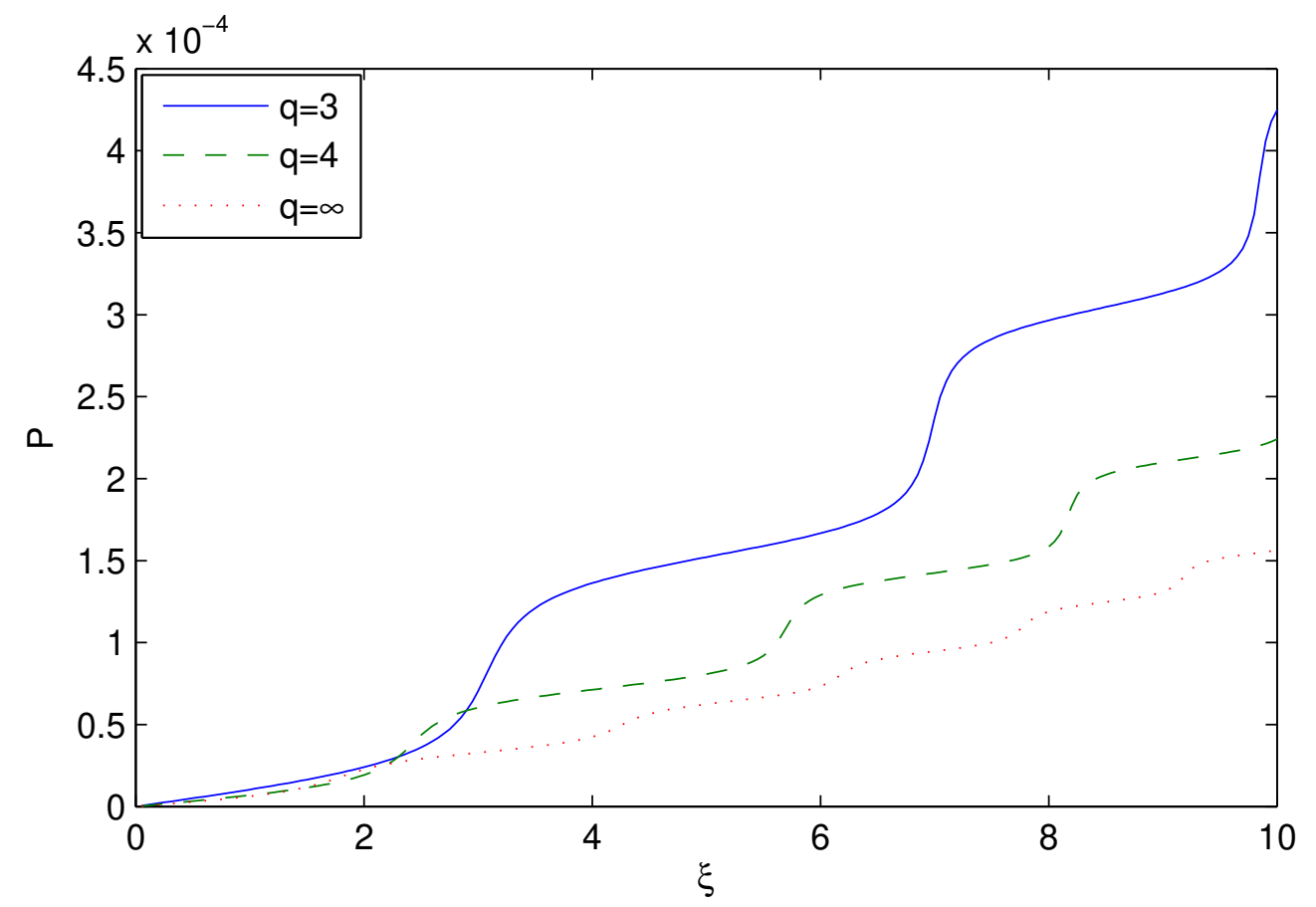

Figure 8: Effect of $\mathrm{q}$ on power of generated second harmonics of the incident laser beam in plasma density ramp.

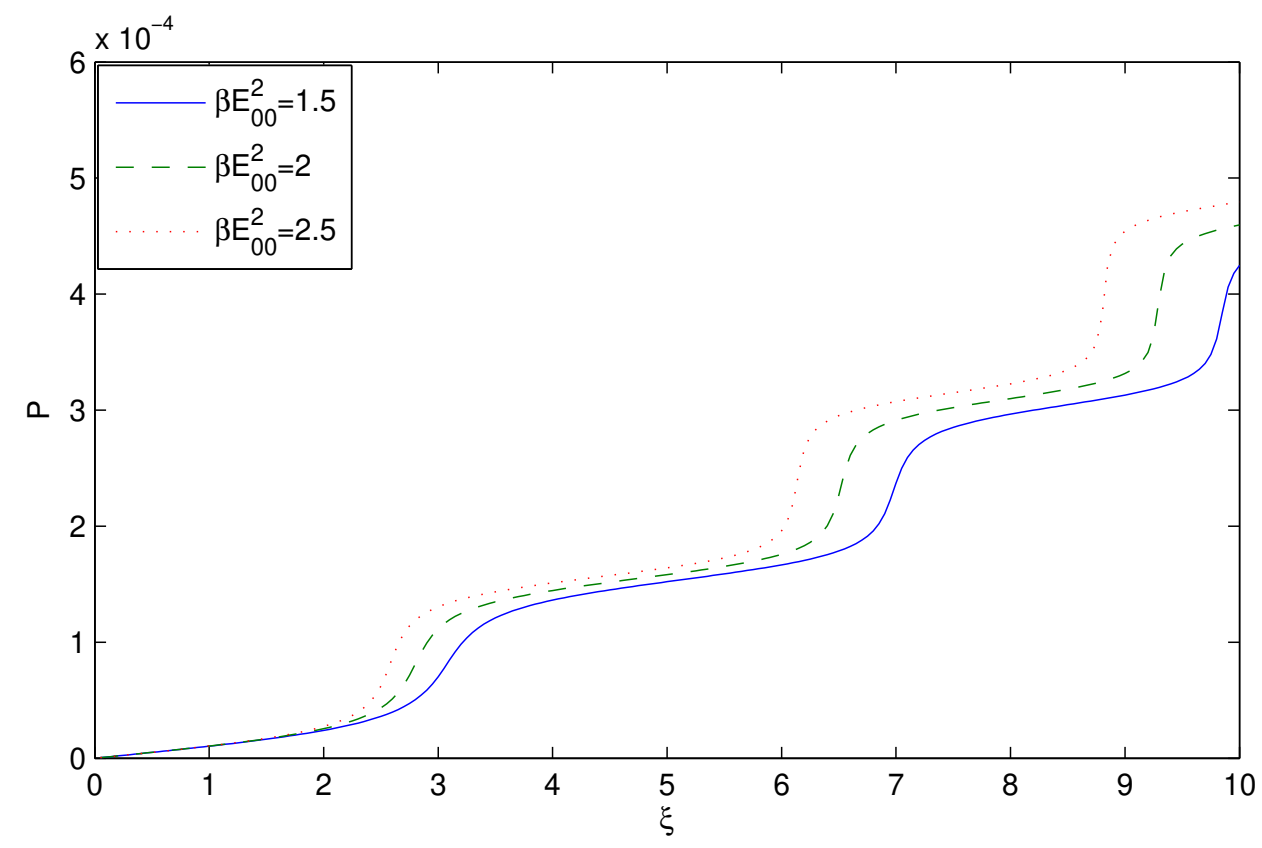

Figure 9: Effect of initial intensity of the laser beam on power of its second harmonics in plasma density ramp. 


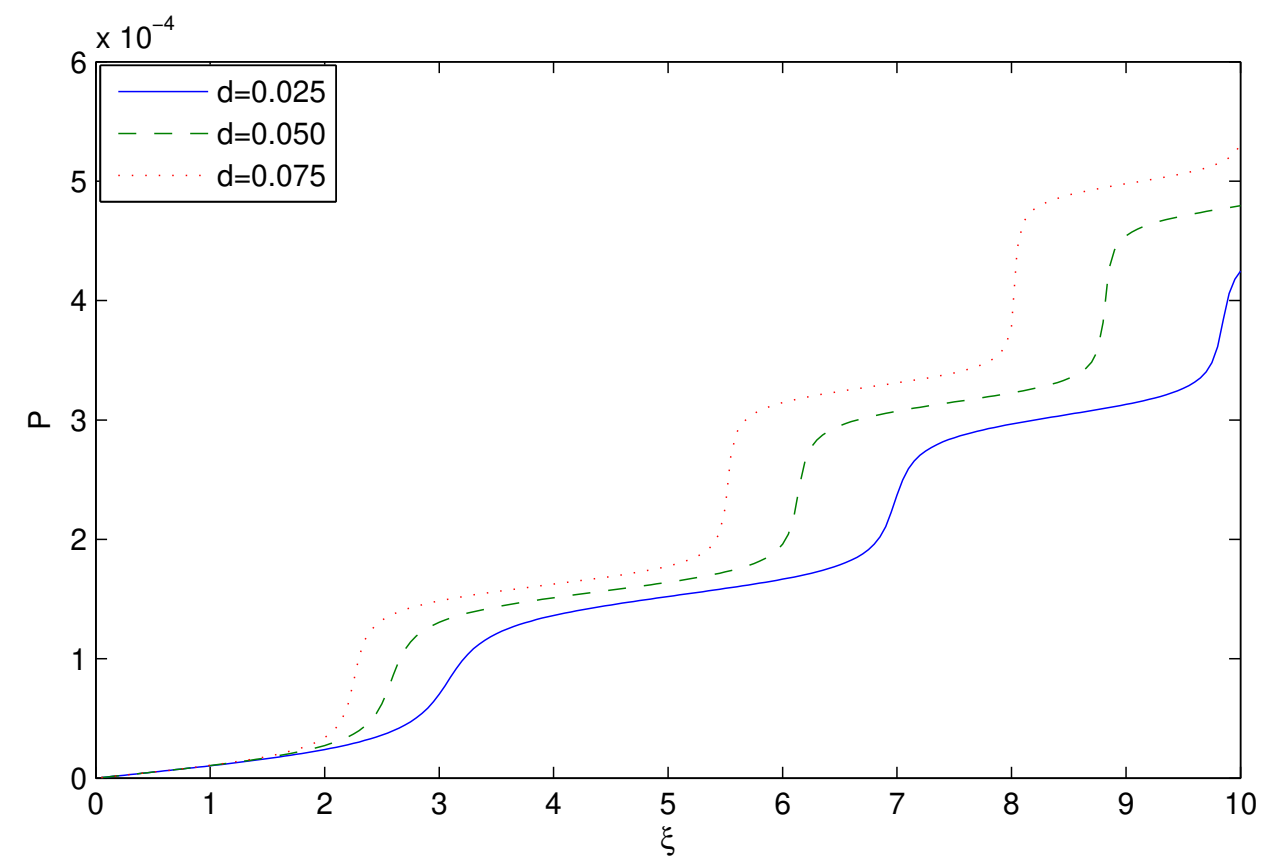

Figure 10: Effect of slope of the density ramp on power of the generated second harmonics of the incident laser beam in plasma density ramp. 
Figures

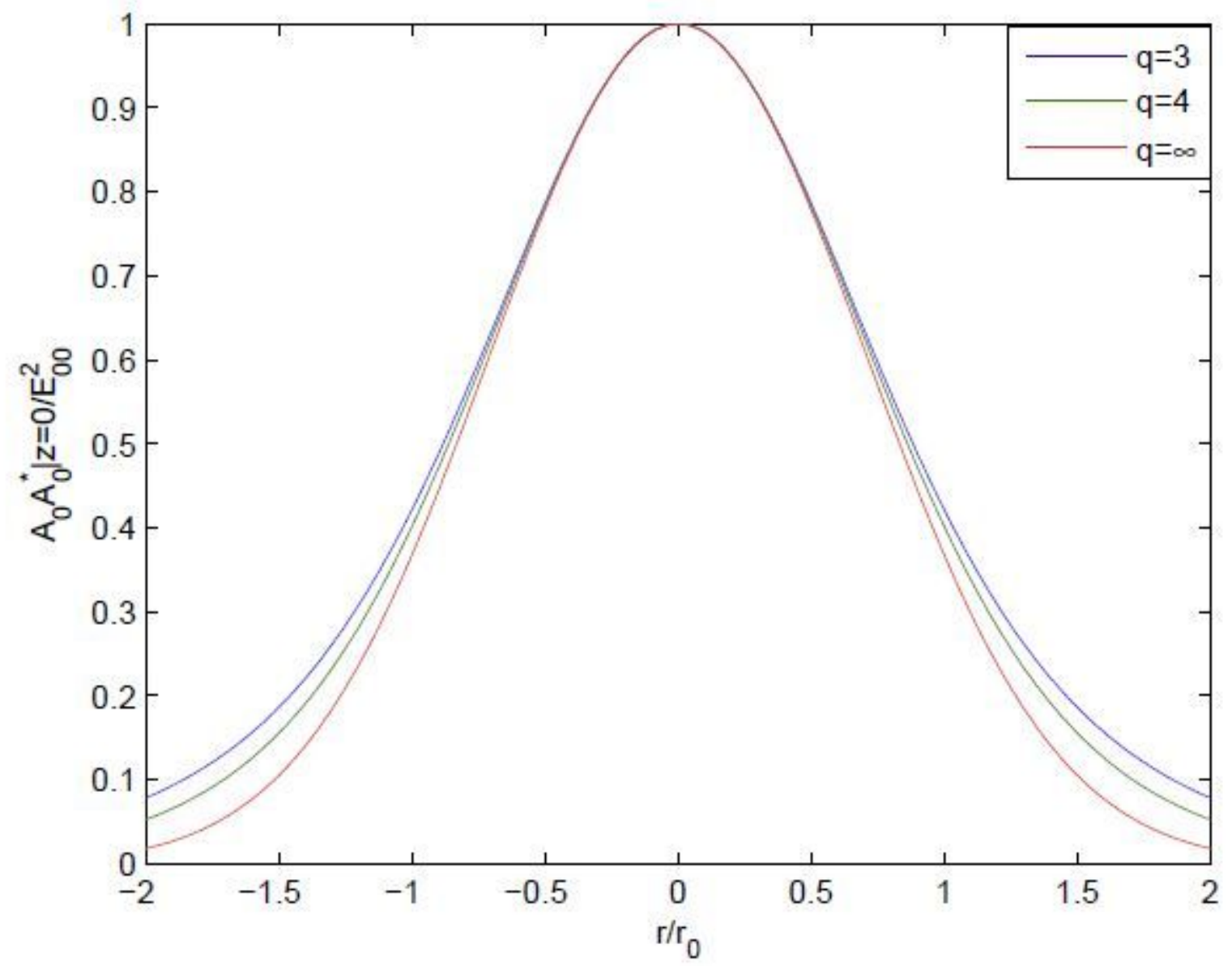

Figure 1

Effect of q on irradiance over the cross section of the laser beam. 18 


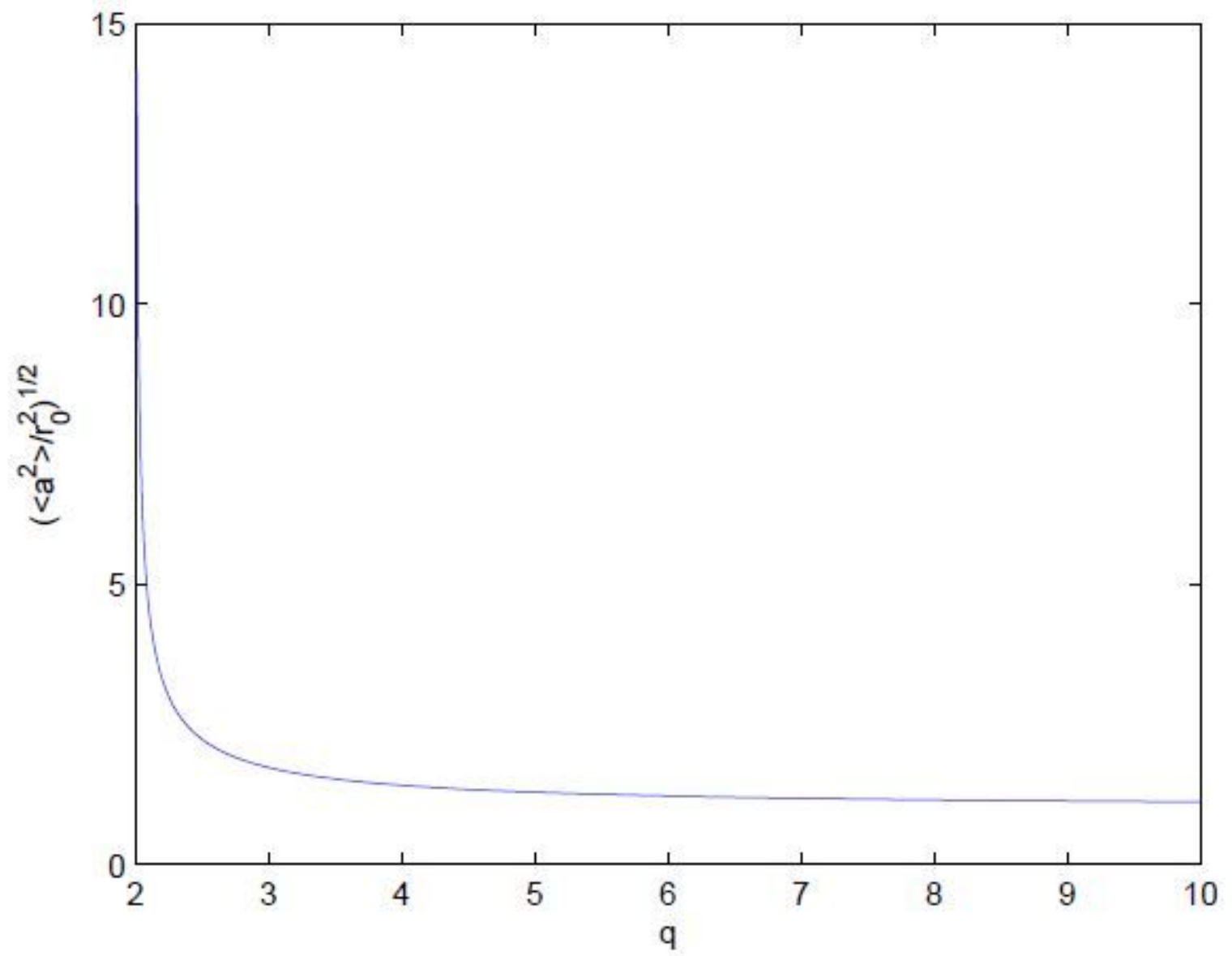

Figure 2

Variation of effective beam width of the laser beam with q. 


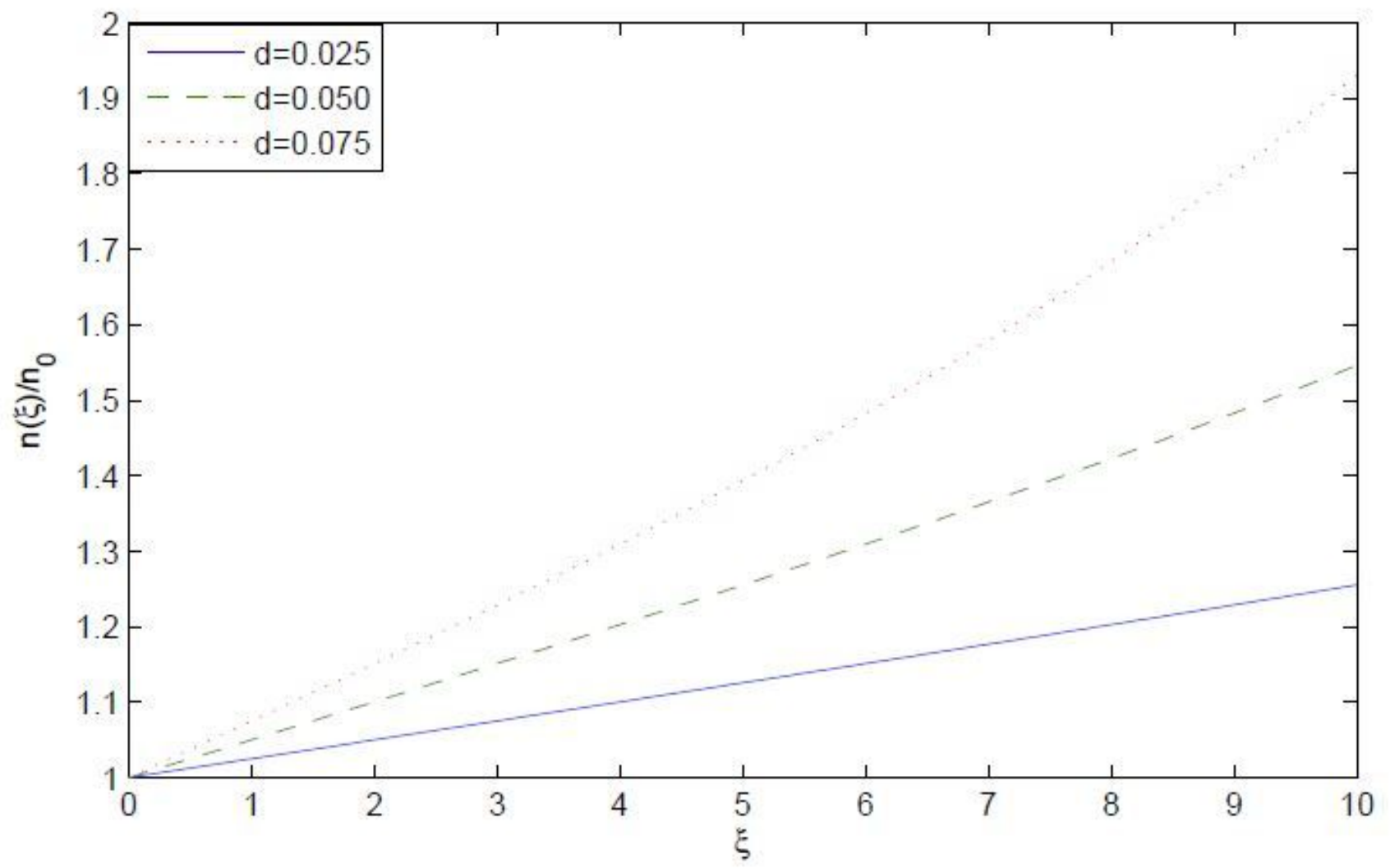

Figure 3

Effect of parameter $\mathrm{d}$ on equilibrium electro density with distance. 


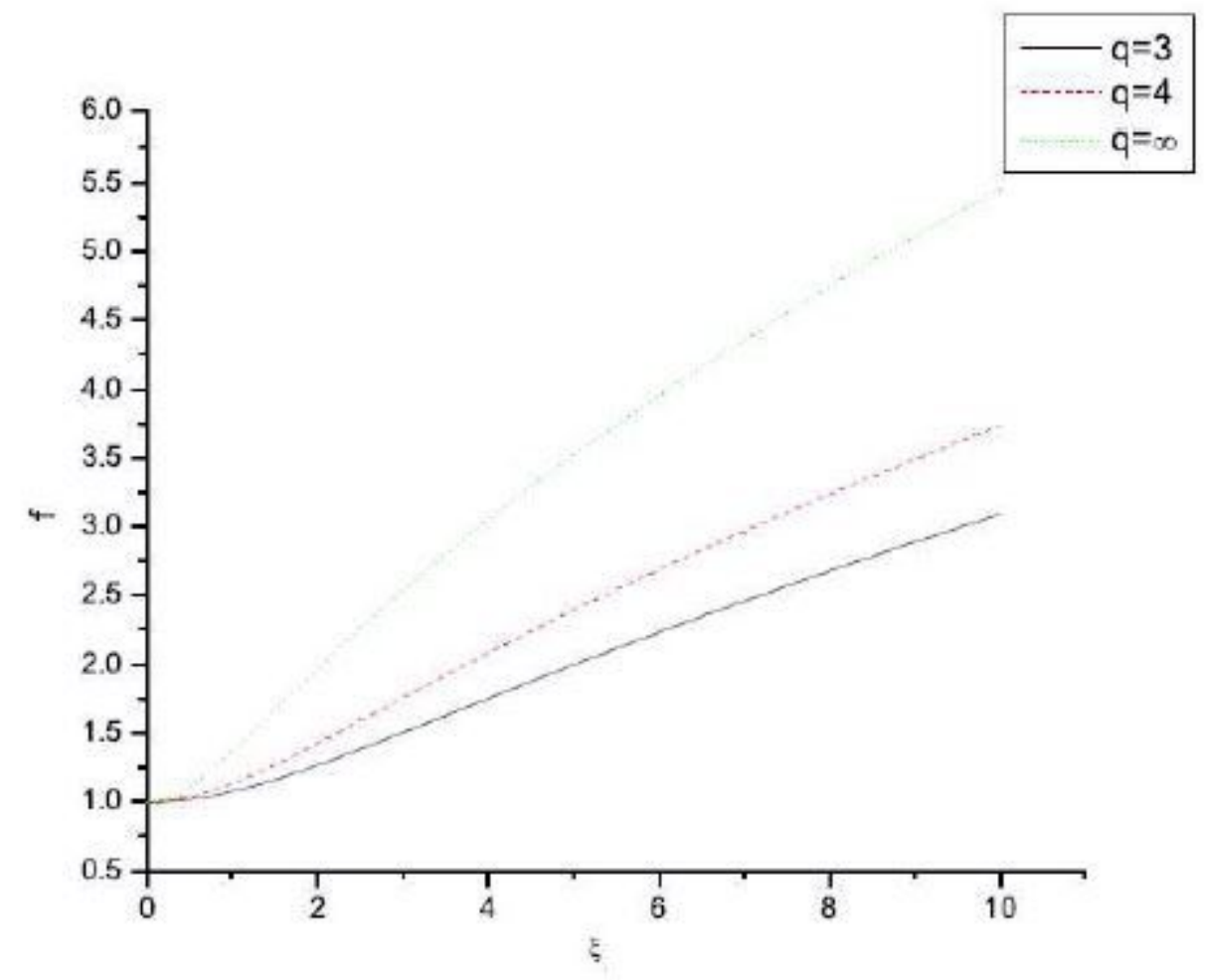

Figure 4

Effect of q on vacuum diffraction of the laser beam. 


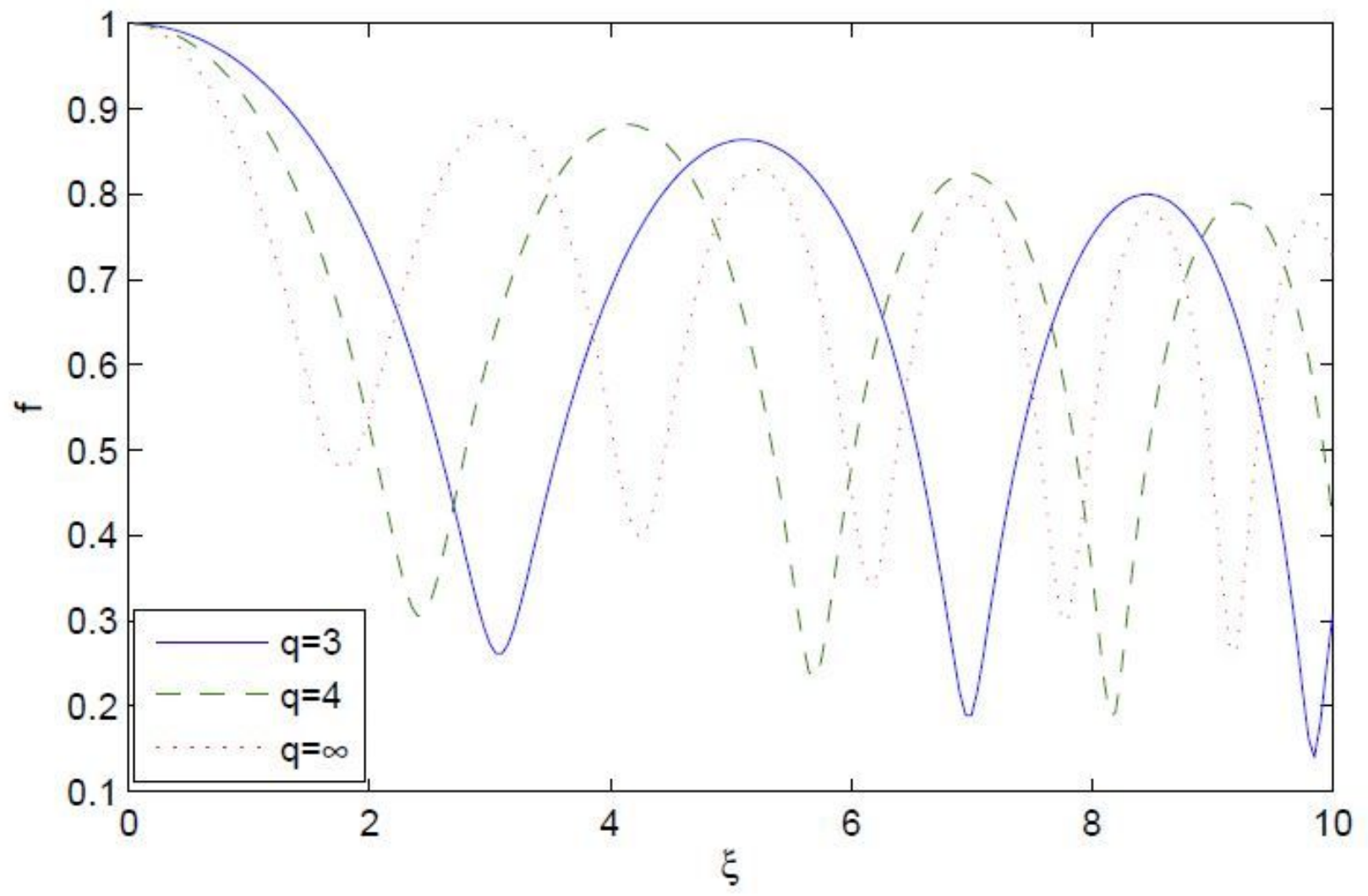

Figure 5

Effect of q on evolution of beam width in plasma density ramp. 


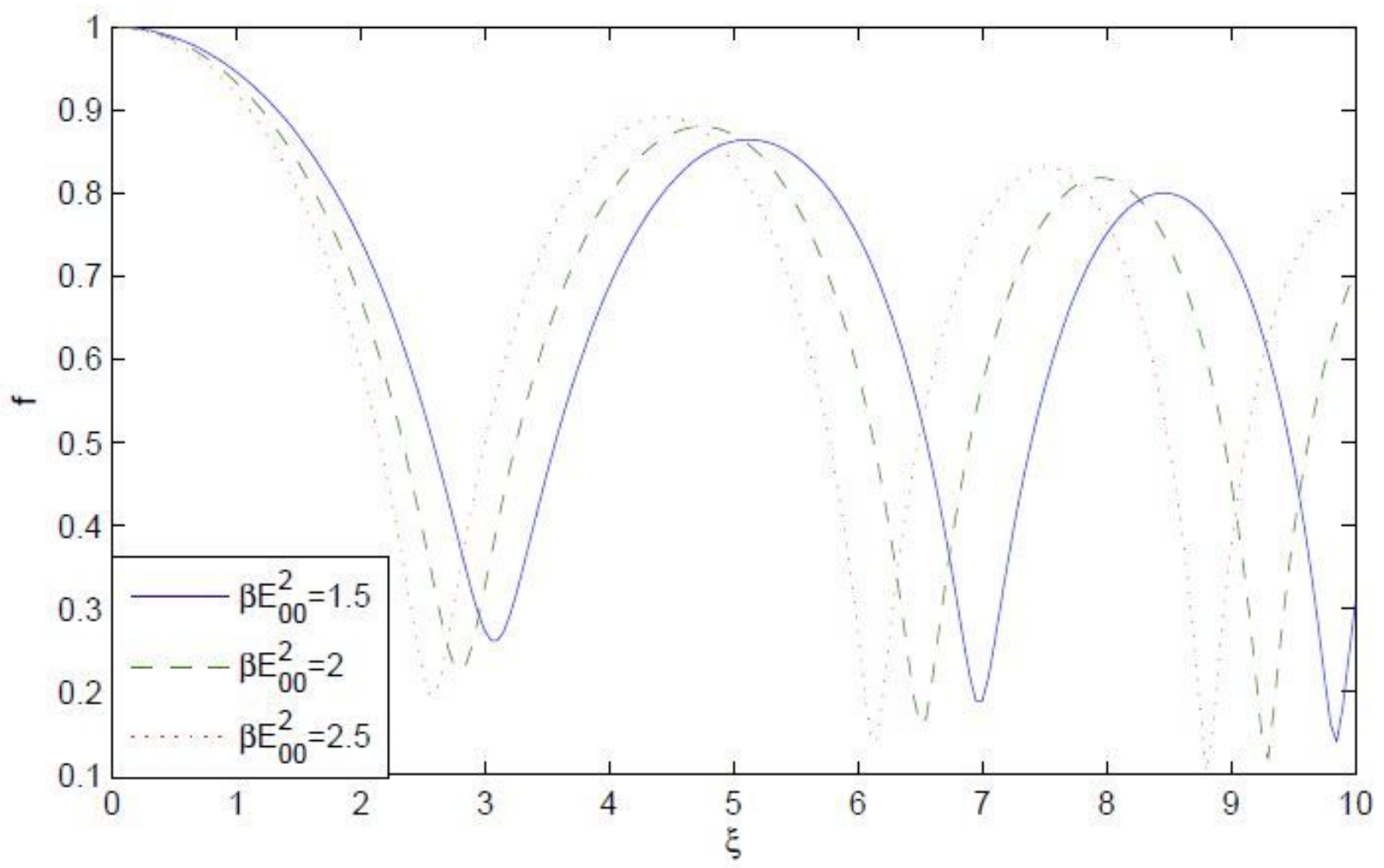

Figure 6

Effect of initial intensity of the laser beam on evolution of its beam width in plasma density ramp. 


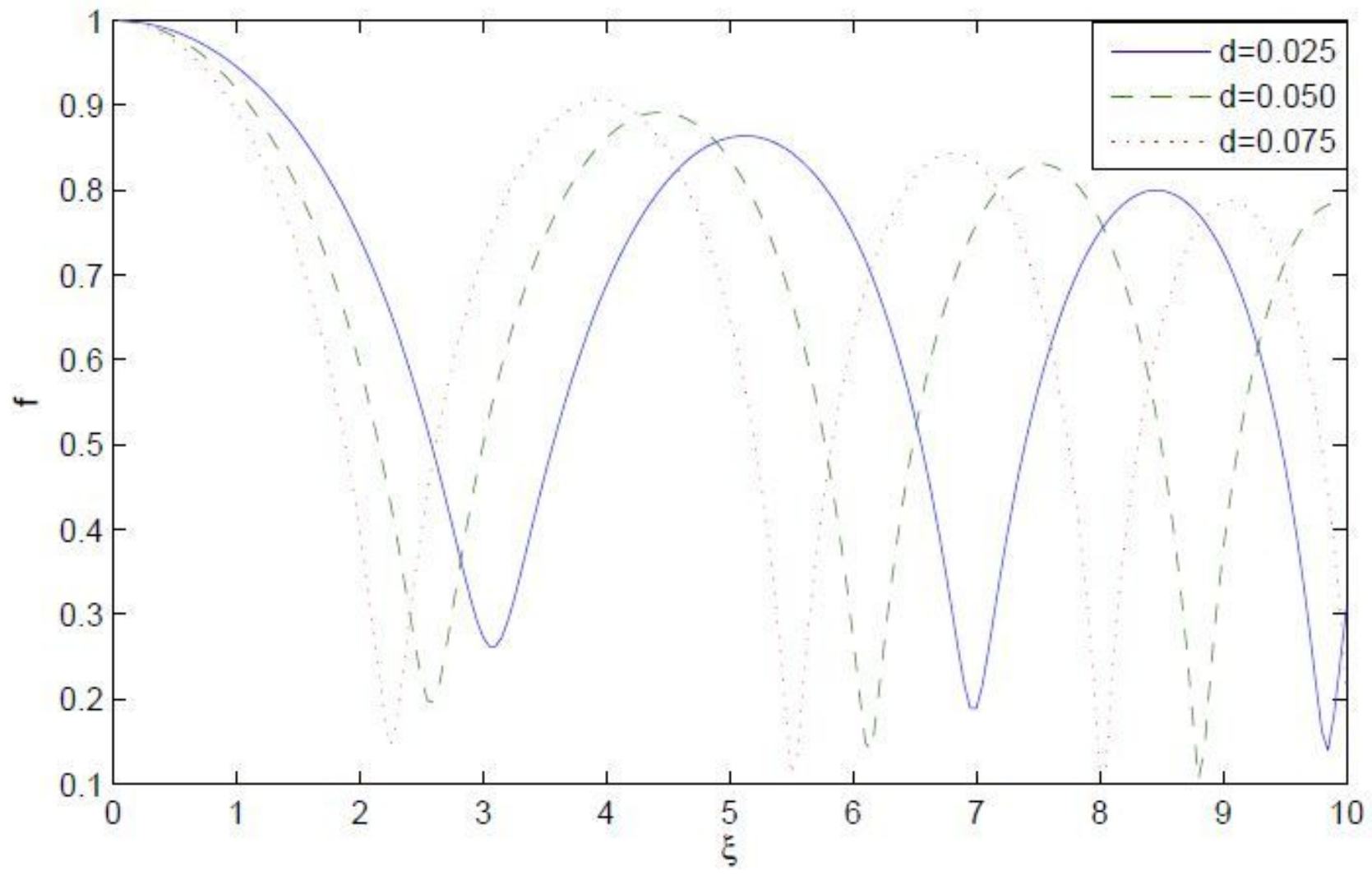

Figure 7

Effect of slope of the density ramp on evolution of beam width of the laser beam in plasma density ramp. 


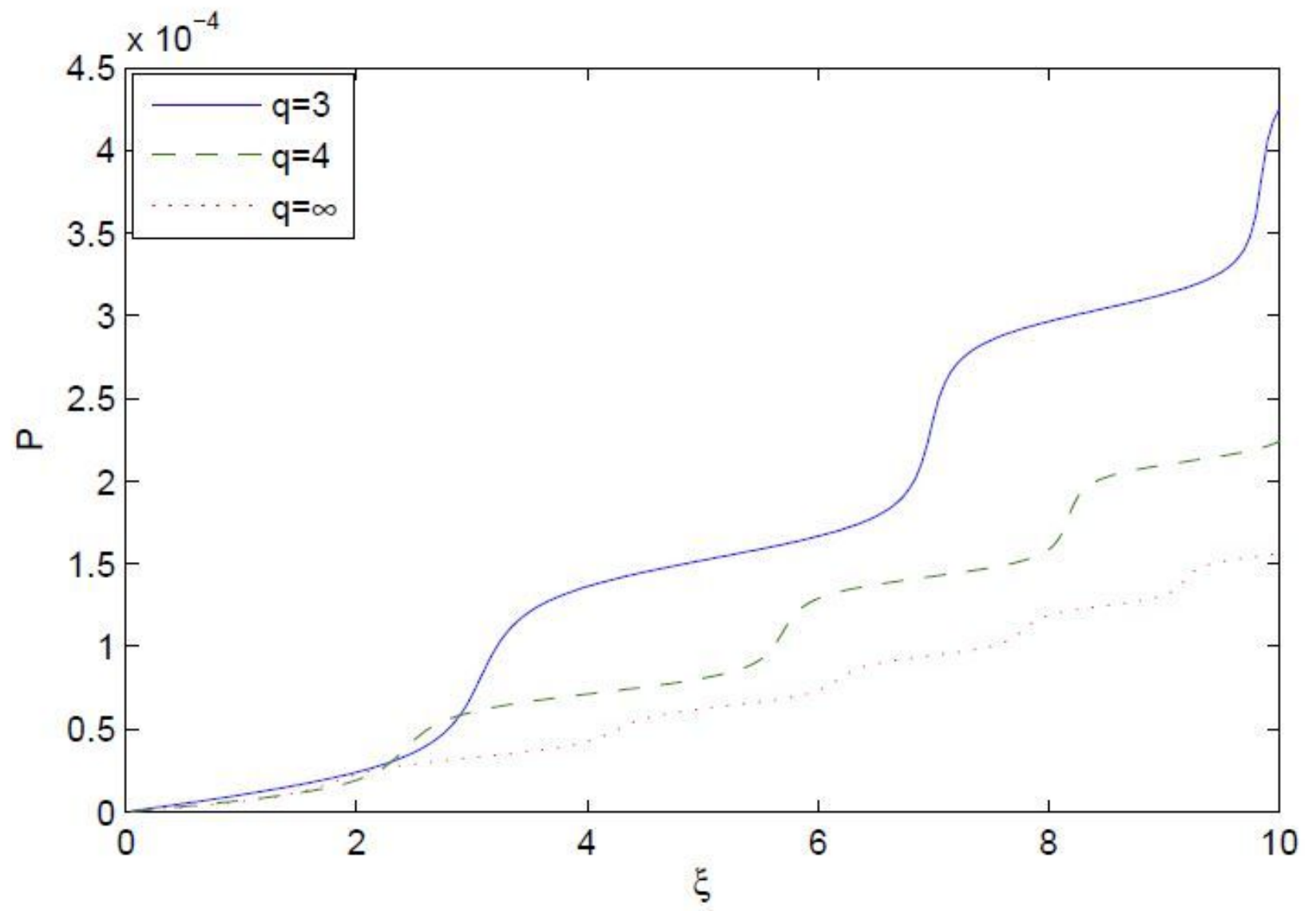

Figure 8

Effect of q on power of generated second harmonics of the incident laser beam in plasma density ramp. 


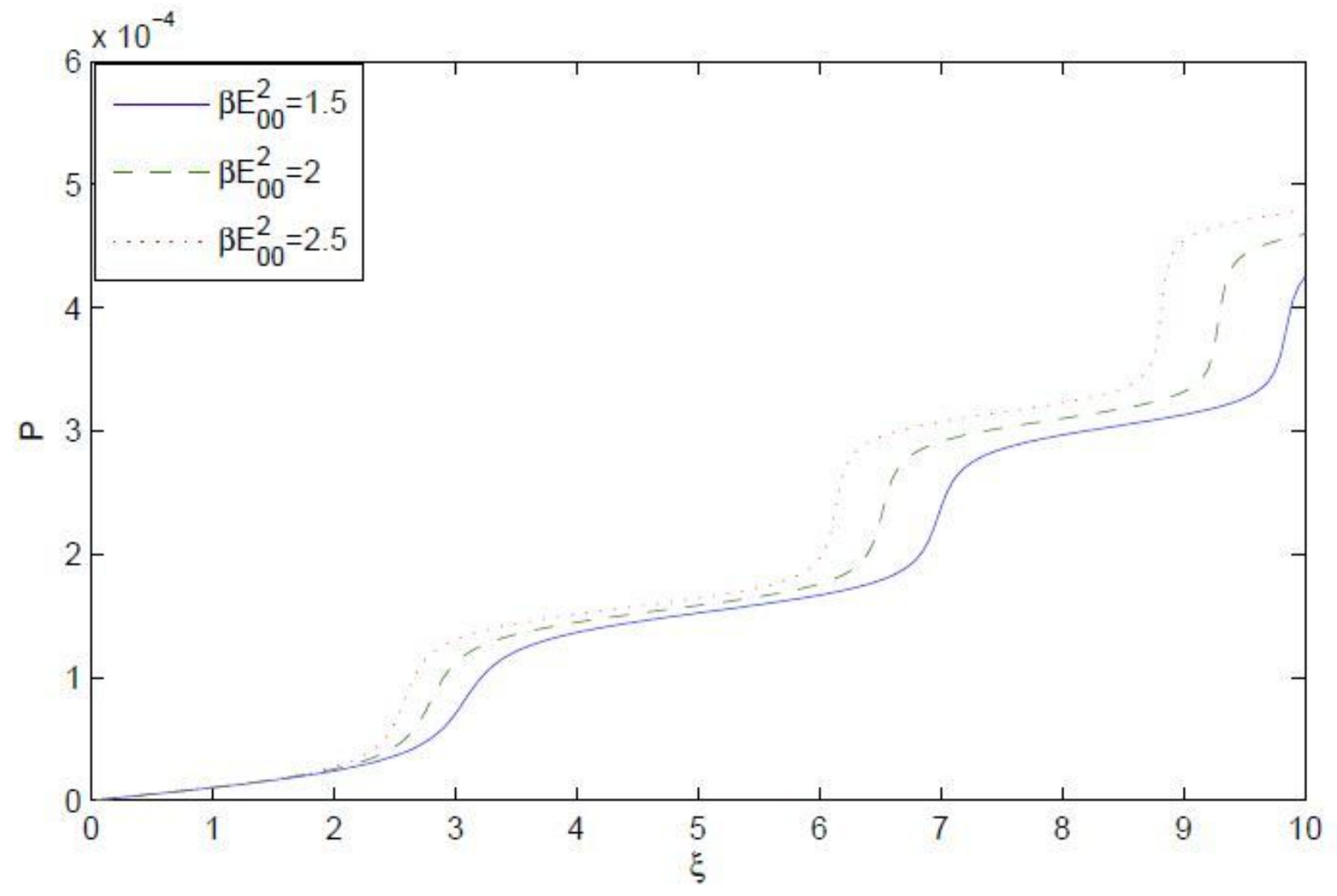

Figure 9

Effect of initial intensity of the laser beam on power of its second harmonics in plasma density ramp. 


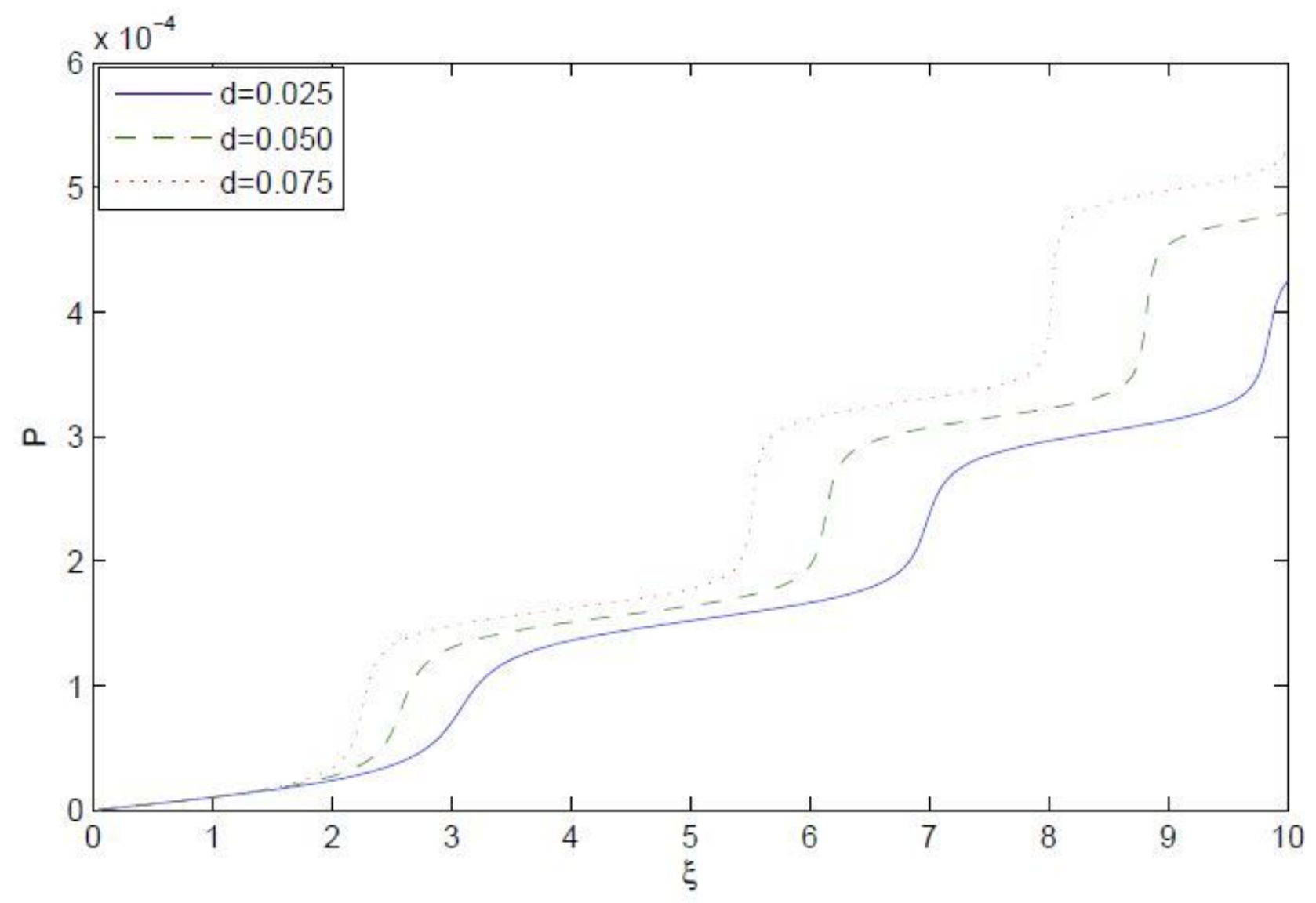

Figure 10

Effect of slope of the density ramp on power of the generated second harmonics of the incident laser beam in plasma density ramp. 